\title{
Videofluoroscopia y disfagia neurogénica
}

Son muchas las enfermedades neurológicas en las que se pueden afectar las estructuras neurales que controlan y dirigen los complicados mecanismos de la deglución orofaríngea (disfagia neurogénica). La prevalencia de alteraciones de la motilidad orofaríngea en pacientes con enfermedades neurológicas y asociadas al envejecimiento es extraordinariamente elevada y muy poco conocida. Más del $30 \%$ de los pacientes que han sufrido un accidente cerebrovascular; el $52-82 \%$ de los pacientes con enfermedad de Parkinson; prácticamente el 100\% de pacientes con esclerosis lateral amiotrófica; $44 \%$ de pacientes con esclerosis múltiple; $84 \%$ de pacientes con enfermedad de Alzheimer, y más del $60 \%$ de ancianos institucionalizados presentan disfagia orofaríngea funcional (1-4). La incidencia de la alteración de la deglución que se produce tras un traumatismo cráneoencefálico (TCE) no ha sido bien estudiada. La bibliografía especifica proporciona una incidencia entre el 25 y el $61 \%$ en pacientes que han sido ingresados para rehabilitación (1-6).

La disfagia orofaríngea funcional tiene un gran impacto social; se estima que en el año 2010, 16.500.000 de jubilados de los EE.UU. requerirán cuidados específicos para su disfagia (7). La severidad del trastorno de la motilidad orofaríngea puede dar lugar a dos grupos de graves complicaciones clínicas: a) hasta el 25$75 \%$ pacientes con disfagia presentan desnutrición y/o deshidratación; y b) el 30-

$50 \%$ presentan aspiración traqueobronquial, que conduce a una neumonía en el $50 \%$ de casos, con una mortalidad asociada de hasta el 50\% $(8,9)$. El progresivo envejecimiento de nuestra sociedad ha causado un incremento del 93,5\% de la tasa de neumonías por aspiración en ancianos en los últimos 10 años, mientras que otros tipos de neumonías han disminuido significativamente $(7,10)$. En la mayoría de nuestros hospitales existe una gran discrepancia entre la elevada morbilidad, mortalidad y elevados costes sanitarios que ocasionan las complicaciones de la disfagia orofaríngea funcional y el bajo nivel de recursos materiales y humanos dedicados a esta patología.

La mayoría de los síntomas y complicaciones que se derivan de una disfagia neurológica se deben a la alteración sensitivo-motora de la fase oral y faríngea de la deglución. Excepcionalmente las alteraciones neurológicas están limitadas exclusivamente a la zona de control deglutorio, por lo que la afectación de otras áreas del sistema nervioso ocasionará otros síntomas neurológicos, que acompañan a la disfagia y complicaran el cuadro clínico. Además algunas actuaciones terapéuticas como la intubación orotraqueal y las cánulas de traqueostomía pueden añadir nuevas dificultades (11), por todo esto el diagnóstico y tratamiento de esta patología requiere un enfoque multidisciplinar con participación de profesionales de enfermería, logopedia, médicos internistas, gastroenterólogos, geriatras, cirujanos digestivos, reha- 
bilitadores, radiólogos, nutricionistas, otorrinolaringólogos, etc. (12). Los principales objetivos de este equipo deben ser: a) la identificación precoz de los pacientes en riesgo de presentar disfagia y/o aspiraciones; b) el diagnóstico de cualquier enfermedad médica o quirúrgica responsable de la disfagia que tenga un tratamiento específico y la exclusión de cualquier causa estructural de disfagia; c) la caracterización de las alteraciones de la respuesta motora orofaríngea mediante exploraciones funcionales como la videofluoroscopia y la manometría faringoesofágica; y d) la selección de las estrategias terapéuticas más apropiadas para lograr una deglución segura y eficaz del paciente, o la indicación de una vía no oral de alimentación -casi siempre una gastrostomía endoscópica percutánea- en base a signos objetivos y reproducibles $(8,9)$.

La disfagia orofaríngea de origen funcional es un síntoma clínicamente poco valorado y poco estudiado a pesar de que existen métodos específicos para su diagnóstico, como la videofluoroscopia y la manometría faringoesofágica (9). La videofluoroscopia (VFS) es la técnica considerada como "patrón de oro" para estudiar los mecanismos orofaríngeos de disfagia funcional $(8,9,13,14)$. La VFS es una exploración radiológica dinámica que permite identificar las principales alteraciones de la deglución en forma de signos videofluoroscópicos (15). Los requisitos técnicos para realizar una VFS son simples -un tubo RX con escopia y un registrador de video-, y disponibles en la gran mayoría de nuestros centros sanitarios (12). Además, se han desarrollado diferentes métodos de análisis asistido por ordenador que permiten la obtención de mediciones cuantitativas espaciales y temporales de la respuesta motora orofaríngea $(16,17)$. Los objetivos de la videofluoroscopia son evaluar la eficacia y la seguridad de la deglución, tipificar las alteraciones de la deglución, evaluar la eficacia de los tratamientos y obtener datos cuantitativos de la biomecánica orofaríngea (15). Los signos VFS que afectan la eficacia de la deglución como la apraxia deglutoria, las alteraciones del control y propulsión lingual, los residuos orofaríngeos y las alteraciones de la apertura del esfínter superior, etc., pueden conducir a una reducción de la alimentación oral y malnutrición. Hasta el 30\% de los pacientes neurológicos y el $80 \%$ de los ancianos "frágiles" presentan malnutrición asociada a la disfagia (15-17). Los principales signos VFS de alteración de la seguridad de la deglución son las penetraciones de contraste en el vestíbulo laríngeo y las aspiraciones (el contraste atraviesa las cuerdas vocales y entra en la vía aérea). Hasta el 40\% de los pacientes con disfagia neurogénica presentan aspiraciones, la mitad de ellas silentes -no acompañadas de tos- y por tanto sólo diagnosticables mediante la aplicación sistemática de esta técnica. La VFS permite determinar el momento del ciclo deglutorio en que se produce la aspiración $-85 \%$ durante la fase faríngea- y también si la aspiración está asociada a una alteración de la "respuesta motora orofaríngea". La respuesta motora orofaríngea durante la deglución es uno de los reflejos digestivos menos conocidos. Comprende dos grandes grupos de acontecimientos: a) la rápida reconfiguración de la orofaringe desde una vía respiratoria en reposo hasta una vía digestiva -básicamente mediante el cierre del vestíbulo laríngeo y la apertura del esfínter esofágico superior-; y b) la intensa propulsión del bolo por la fuerza de la lengua $(7,8,17)$. La VFS permite identificar y cuantificar la mayoría de estos elementos. Recientes estudios han demostrado que la alteración de la fuerza de propulsión lingual es la principal responsable de una deglución ineficaz (17) y que las principales alteraciones responsables de las aspiraciones son el enlentecimento de la fase inicial de la reconfiguración orofaríngea $(17,18)$, y la alteraciones de los mecanismos de protección de la vía aérea (elevación hioidea y laríngea, descenso epiglótico y cierre de cuerdas vocales) (8). Por otro lado, la evaluación de los mecanismos 
de apertura del esfínter superior requiere en ocasiones de estudios combinados mediante VFS y manometría. La manometría permite valorar la presión intrabolo (indirectamente la complianza), la relajación (pero no la apertura) y la contracción del EES, mientras que VFS informará de los movimientos de tracción hioidea, de al apertura del EES y de la propulsión lingual (19). Los mejores resultados de la indicación de miotomía del cricofaríngeo se obtienen en aquellos pacientes que presentan una apertura incompleta en la VFS y una disminución de la complianza del esfínter en la manometría y que además presentan integridad en la respuesta motora orofarígea (reconfiguración orofaríngea rápida, propulsión lingual vigorosa y movimiento hioideo amplio) (19). Los avances en tecnología médica proporcionarán en el futuro inmediato métodos de estudio de la fisiología orofaríngea basados en la obtención automatizada de parámetros videofluorscópicos y la introducción de nuevas herramientas como la manometría de alta definición.

Este número de la Revista Española de Enfermedades Digestivas incluye un interesante estudio sobre la cuantificación videofluoroscópica de la disfagia orofaríngea secundaria a lesión cerebral traumática. En él, los autores establecen la utilidad clínica de la videofluoroscopia, demuestran la alta frecuencia con la que se producen alteraciones de la deglución en pacientes con TCE grave, $65 \%$ en la fase oral y $73 \%$ en la faríngea, identifican un $90 \%$ de pacientes con aspiraciones durante la fase faríngea -la mayoría silentes, no identificables clínicamente- a pesar de la preservación en estos pacientes de los parámetros cuantitativos que definen la fase faríngea de la deglución (20). A diferencia de los pacientes con disfagia asociada a enfermedades neurodegenerativas y al envejecimiento, la evolución natural de la disfagia en los pacientes con TCE es favorable; no obstante hay estudios que confirman que la presencia de una alteración en la deglución alarga la estancia hospitalaria (21).

Está demostrado que la aplicación de programas terapéuticos a los pacientes con disfagia orofaríngea se correlaciona con una importante reducción en las tasas de neumonía aspirativa y en la mejoría del estado nutricional. El tratamiento puede incluir cambios en volumen y la viscosidad del bolo, estrategias posturales, praxias orofaríngeas y maniobras activas, y procedimientos de la rehabilitación $(9,22)$. El nivel más alto de evidencia terapéutica corresponde al incremento de la viscosidad del bolo alimentario, que ocasiona una reducción tan dramática de las aspiraciones que incluso se ha recomendado su utilización de forma sistemática en los pacientes en riesgo de aspiración (8-10,16). En los pacientes con alteraciones de la eficacia de la deglución debe ajustarse sus requerimientos calóricos y proteicos al volumen de alimento que puedan deglutir para evitar riesgos nutricionales. Una reciente resolución del Consejo de Europa sobre el cuidado nutricional en nuestros hospitales enfatiza el efecto negativo de la malnutrición en la prolongación de las estancias hospitalarias y las necesidades de rehabilitación, en la reducción de la calidad de vida y en el incremento de los costes sanitarios, y recomienda considerar la disfagia orofaríngea funcional como un importante factor de riesgo nutricional (23). Muy recientes estudios sugieren el inmediato desarrollo de herramientas farmacológicas para la estimulación de la respuesta motora orofaríngea.

El reconocimiento de la disfagia orofaríngea funcional como un síndrome neurológico y geriátrico específico inducirá muchos cambios en la provisión de servicios médicos y sociales en un futuro próximo. La educación de los profesionales de salud en el diagnóstico y el tratamiento de la disfagia y sus complicaciones, el desarrollo de métodos clínicos de screening para su diagnóstico precoz, el desarrollo de exploraciones complementarias específicas en el ámbito clínico, el desarrollo en las estrategias dietéticas para evitar aspiraciones y la desnutrición, y la investigación de 
la fisiopatología, farmacología e historia natural de la disfagia en cada enfermedad son las piedras angulares necesarias para permitir el máximo potencial de recuperación para los pacientes con disfagia orofaríngea funcional.

\author{
A. Ruiz de León y P. Clavé1 \\ Servicio de Aparato Digestivo. Hospital Clínico San Carlos. Madrid. \\ ${ }^{1}$ Unidad de Exploraciones Funcionales Digestivas. Hospital de Mataró. Barcelona
}

\title{
BIBLIOGRAFÍA
}

1. Winstein CJ. Neurogenic dysphagia. Frequency, progression, and outcome in adults following head injury. Phys Ther 1983; 63 (12): 1992-7.

2. Mackay LE, Morgan AS, Bernstein BA. Swallowing disorders in severe brain injury: risk factors affecting return to oral intake. Arch Phys Med Rehabil 1999; 80 (4): 365-71.

3. Lazarus C, Logemann JA. Swallowing disorders in closed head trauma patients. Arch Phys Med Rehabil 1987; 68 (2): 79-84.

4. Field LH, Weiss CJ. Dysphagia with head injury. Brain Inj 1989; 3 (1): 19-26.

5. Meadows J. Dysphagia in unilateral cerebral lesions. J Neurol Neurosurg Psychiatry 1993; 36: 853-60.

6. Horner J, Massey EW. Managing dysphagia: Special problems in patients with neurologic disease. Dysphagia 2001; 89: 203.

7. Robbins J, Langmore S, Hind JA, Erlichman MS. Dysphagia research in the 21st century and beyond: Proceedings from dysphagia experts Meeting, August 21, 2001. Journal of Rehabilitation Research Development 2002; 39: 543-8.

8. Cook IJ, Kahrilas PJ. AGA technical review on management of oropharyngeal dysphagia. Gastroenterology 1999; 116: 455-78.

9. Clave P, Terre R, de Kraa M, Serra M. Approaching oropharyngeal dysphagia. Rev Esp Enferm Dig 2004; 96: 119-31.

10. Clavé P, Verdaguer A, Arreola V. Oral-pharyngeal dysphagia in the elderly. Med Clin (Barc) 2005; 124(19): $742-8$.

11. Tolep K, Getch CL, Criner GJ. Swallowing dysfunction in patients receiving prolonged mechanical ventilation. Chest 1996; 109 (1): 167-72.

12. Clavé P, Almirall J, Esteve M, Verdaguer A, Berenguer J, Serra-Prat M. Oropharyngeal dysphagia. A team approach to prevent and treat complications. Hospital Healthcare Europe 2005/2006. Campden Publishing Ltd (eds). London N5-N8, 2005.

13. Clavé P. Métodos de estudio de la neurofisiología de la deglución y de la disfagia orofaríngea. Rev Esp Enferm Dig 2004; 96 (Supl. II): 47-9.

14. Clavé P. Videofluoroscopic diagnosis of oropharyngeal dysphagia. Nutrition Matters 2001; 3: 1-2.

15. Clavé P. Diagnóstico de la disfagia neurógena. Exploraciones complementarias en disfagia neurógena: evaluación y tratamiento. Blocs 14. Barcelona: Fundació Institut Guttmann (ed); 2002. p. 19-27.

16. Logemann JA, Kahrilas PJ, Begelman J, Dodds WJ, Pauloski BR. Interactive computer program for biomechanical analysis of videoradiographic studies of swallowing. AJR Am J Roentgenol 1989; 153: 277-80.

17. Clavé P, de Kraa M, Arreola V, Girvent M, Farré R, Palomera E, et al. The effect of bolus viscosity on swallowing function in neurogenic dysphagia. Aliment Pharmacol Ther 2006; 24: 1385-94.

18. Kahrilas PJ, Lin S, Rademaker AW, Logemann JA. Impaired deglutitive airway protection: a videofluoroscopic analysis of severity and mechanism. Gastroenterology 1997; 113: 1457-64.

19. Williams RBH, Wallace KL, Ali GA, Cook IJ. Biomechanics of failed deglutitive upper esophageal sphincter relaxation in neurogenic dysphagia. Am J Physiol (Gastrointestinal Liver Physiol) 2002; 283: G16-G26.

20. Terré R, Mearín F. Evolución de la aspiración laringo-traqueal en la disfagia orofaríngea secundaria a lesión cerebral traumática: cuantificación videofluoroscópica. Rev Esp Enferm Dig 2007; 99 (1): 7-12.

21. Kidd D, Lawson, Nesbitt R, et al. Aspiration in acute a stroke: A clinical study with videofluroscopy. Q J Med 1993; 86: 825-9.

22. Logemann JA. Dysphagia - Evaluation and Treatment. Folia Phoniatrica et Logopaedica 1995; 47: 140-64.

23. Council of Europe Committee of Ministers. Resolution on Food and Nutritional Care in Hospitals. Available at: $w w w . w c m . c o e . i n t / V i e w D o c . j s p ? i d=85747 \&$ Lang=en, 2003. 